\title{
Steroid-Refractory Pneumonitis
}

National Cancer Institute

\section{Source}

National Cancer Institute. Steroid-Refractory Pneumonitis. NCI Thesaurus. Code C156671.

Pneumonitis that does not respond to corticosteroid therapy. 